\title{
Which medical disciplines diagnose and treat melanoma in Europe in 2019? A survey of experts from melanoma centres in 27 European countries
}

L. Kandolf-Sekulovic, ${ }^{1,{ }^{*}}$ (D) K. Peris, ${ }^{2}$ (D) A. Stratigos, ${ }^{3}$ A. Hauschild, ${ }^{4}$ A.-M. Forsea, ${ }^{5}$ (D) C. Lebbe, ${ }^{6}$ A. Lallas, ${ }^{7}$ iD J.-J. Grob, ${ }^{8}$ iD C. Harwood, ${ }^{9}$ H. Gogas, ${ }^{10}$ P. Rutkowski, ${ }^{11}$ J. Olah, ${ }^{12}$ N.W.J. KellenersSmeets, ${ }^{13}$ J. Paoli, ${ }^{14}$ (iD R. Dummer, ${ }^{15}$ (iD D. Moreno-Ramirez, ${ }^{16}$ L. Bastholt, ${ }^{17}$ K. Putnik, ${ }^{18}$ R. Karls,${ }^{19}$ C. Hoeller, ${ }^{20}$ V. Vandersleyen, ${ }^{21}$ R. Vieira,${ }^{22}$ P. Arenberger, ${ }^{23}$ M. Bylaite-Buckinskiene,${ }^{24} \mathrm{~J}$. Ocvirk, ${ }^{25}$ M. Situm, ${ }^{26}$ G. Weinlich, ${ }^{27}$ M. Banjin, ${ }^{28}$ V. Todorovic, ${ }^{29}$ A. Ymeri, ${ }^{30}$ A. Zhukavets, ${ }^{31}$ C. Garbe ${ }^{32}$

${ }^{1}$ Department of Dermatology, Faculty of Medicine, Military Medical Academy, Belgrade, Serbia

${ }^{2}$ Institute of Dermatology, Catholic University of the Sacred Heart, Rome, Italy

${ }^{3}$ 1st Department of Dermatology-Venereology, Andreas Sygros Hospital, National and Kapodistrian University of Athens, Athens, Greece

${ }^{4}$ Department of Dermatology, University Hospital Schleswig-Holstein (UKSH), Kiel, Germany

${ }^{5}$ Elias University Hospital Bucharest, Carol Davila University of Medicine and Pharmacy, Bucuresti, Romania

${ }^{6}$ APHP Dermatology Department, University Paris 7 Diderot, INSERM U976, Paris, France

${ }^{7}$ First Department of Dermatology, Aristotle University, Thessaloniki, Greece

${ }^{8}$ Service de Dermatologie et Cancérologie Cutanée, Hopital de la Timone, Marseille, France

${ }^{9}$ Centre for Cell Biology and Cutaneous Research, Blizard Institute, Barts and the London School of Medicine and Dentistry, Queen

Mary University of London, London, UK

${ }^{10} 1$ st Department of Internal Medicine, Laiko Hospital, National and Kapodistrian University of Athens, Athens, Greece

${ }^{11}$ Maria Sklodowska-Curie Institute - Oncology Center, Warsaw, Poland

${ }^{12}$ Department of Oncotherapy, Department of Dermatology and Allergology, University of Szeged, Szeged, Hungary

${ }^{13}$ Department of Dermatology, Maastricht University Medical Center, Maastricht, the Netherlands

${ }^{14}$ Department of Dermatology and Venereology, Institute of Clinical Sciences, Sahlgrenska Academy, University of Gothenburg,

Gothenburg, Sweden

${ }^{15}$ UniversitätsSpital Zürich-Skin Cancer Center, University Hospital, Zürich, Switzerland

${ }^{16}$ Department of Clinical Oncology, Hospital Universitario Virgen Macarena, Sevilla, Spain

${ }^{17}$ Department of Oncology, Odense University Hospital, Odense, Denmark

${ }^{18}$ North Estonia Medical Centre, Tallinn, Estonia

${ }^{19}$ Derma Clinic Riga, Riga, Latvia

${ }^{20}$ Department of Dermatology, Medical University of Vienna, Vienna, Austria

${ }^{21}$ Department of Medical Oncology, Universitair Ziekenhuis Brussel, Vrije Universiteit Brussel (VUB), Brussels, Belgium

${ }^{22}$ Department of Dermatology, Medical Faculty, University of Coimbra, Coimbra, Portugal

${ }^{23}$ Department of Dermatovenereology, Charles University 3rd Faculty of Medicine and University Hospital Kralovske Vinohrady, Prague, Czech Republic

${ }^{24}$ Department of Dermatology, Vilnius University, Vilnius, Lithuania

${ }^{25}$ Institute of Oncology Ljubljana, Ljubljana, Slovenia

${ }^{26}$ Department of Oncology, University Hospital Zagreb, Zagreb, Croatia

${ }^{27}$ Department of Dermatology, Venerology and Allergology, Medical University of Innsbruck, Innsbruck, Austria

${ }^{28}$ Department of Oncology, University Hospital Sarajevo, Sarajevo, Bosnia and Herzegovina

${ }^{29} \mathrm{Clinic}$ for Oncology and radiotherapy, Podgorica, Montenegro

${ }^{30}$ University Hospital Mother Theresa, Tirana, Albania

${ }^{31}$ Belarusian Medical Academy of Postgraduate Education (BelMAPE), Minsk, Belarus

${ }^{32}$ Centre for Dermatooncology, Department of Dermatology, Eberhard Karls University, Tuebingen, Germany

*Correspondence: L. Kandolf Sekulovic. E-mail: Ikandolfsekulovic@gmail.com

\section{Abstract}

Background and objectives The incidence of melanoma is increasing. This places significant burden on societies to provide efficient cancer care. The European Cancer Organisation recently published the essential requirements for quality melanoma care. The present study is aimed for the first time to roughly estimate the extent to which these requirements have been met in Europe. 
Materials and methods A web-based survey of experts from melanoma centres in 27 European countries was conducted from 1 February to 1 August 2019. Data on diagnostic techniques, surgical and medical treatment, organization of cancer care and education were collected and correlated with national health and economic indicators and mortalityto-incidence ratio (MIR) as a surrogate for survival. Univariate linear regression analysis was performed to evaluate the correlations. SPSS software was used. Statistical significance was set at $P<0.05$.

Results The MIR was lower in countries with a high health expenditure per capita and with a higher numbers of general practitioners (GPs) and surgeons (SURG) per million inhabitants. In these countries, GPs and dermatologists (DER) were involved in melanoma detection; high percentage of DER used dermatoscopy and were involved in the follow-up of all melanoma stages; both medical oncologists (ONC) and dermato-oncologists administered systemic treatments; and patients had better access to sentinel lymph node biopsy and were treated within multidisciplinary tumour boards.

Conclusion Based on these first estimates, the greater involvement of GPs in melanoma detection; the greater involvement of highly trained DER in dermatoscopy, dermatosurgery, follow-up and the systemic treatment of melanoma; and the provision of ongoing dermato-oncology training for pathologists, SURG, DER and ONC are necessary to provide an optimal melanoma care pathway. A comprehensive analysis of the melanoma care pathway based on clinical melanoma registries will be needed to more accurately evaluate these first insights.

Received: 7 April 2020; Accepted: 20 November 2020

\section{Conflicts of interest}

Authors declare no relevant conflict of interest for any aspect of submitted work. Relevant financial activities outside the submitted work are listed. LKS reports personal fees and non-financial support from MSD, and personal fees from Novartis, Roche, BMS and AbbVie, outside the submitted work. KP reports personal fees from Almirall, AbbVie, Biogen, Eli Lilly, Celgene, Galderma, Leo Pharma, Novartis, Pierre Fabre, Sanofi, Sandoz, Sun Pharma and Janssen, outside the submitted work. AS reports personal fees and/or research support from Novartis, Roche, BMS, AbbVie, Sanofi, Regeneron and Genesis Pharma outside the submitted work. AH reports grants and personal fees from BMS, Novartis Pharma, Merck Serono, MSD/Merck, Philogen, Pierre Fabre, Provectus, Regeneron, Roche and Sanofi Genzyme, and personal fees from Amgen, OncoSec, Almirall Hermal and Sun Pharma, outside the submitted work. AMF reports personal fees from Merck, Novartis and Solartium, and non-financial support from Leo Pharma and La Roche-Posay, outside the submitted work. CL reports grants and personal fees from Bristol Myers Squibb and Roche, and personal fees from MSD, Novartis, Amgen, Avantis Medical Systems, Pierre Fabre, Pfizer, Incyte, Merck Serono and Sanofi, outside the submitted work. JJG reports personal fees and non-financial support from BMS, Novartis and MSD, and personal fees from Roche, Amgen, Pierre Fabre, Merck, Pfizer and Sanofi, outside the submitted work; HG reports grants and personal fees from BMS, Roche and MSD, and personal fees from Novartis, Amgen and Pierre Fabre, outside the submitted work. PR reports personal fees from Novartis, MSD, BMS, Roche, Pfizer, Blueprint Medicines and Pierre Fabre, outside the submitted work. NKS reports personal fees from Janssen, Galderma and AbbVie, outside the submitted work. RD reports grants and personal fees from Novartis, MSD, BMS, Roche, Amgen, Takeda, Pierre Fabre, Sun Pharma, Sanofi, CatalYm and Second Genome outside the submitted work. LB reports personal fees from advisory boards BMS, Novartis and Merck/MSD, and personal fees from Swedish Orphan, Bayer and Incyte, outside the submitted work. KP reports grants and personal fees from Almirall and AbbVie, and personal fees from Biogen, Lilly, Celgene, Galderma, Leo Pharma, Novartis, Pierre Fabre, Sanofi, Sandoz, Sun Pharma and Janssen, outside the submitted work. $\mathrm{CH}$ reports personal fees from MSD, Roche, Novartis, BMS and Pierre Fabre, outside the submitted work. JO reports personal fees from Roche, Merck, MSD, Lilly, BMS, Sanofi and Novartis, outside the submitted work. VT reports personal fees and non-financial support from Roche, Novartis, Sanofi, Astra Zeneca, MSD and Merck, outside the submitted work. CG reports grants and personal fees from Novartis, NeraCare, BMS, Roche, Philogen and Sanofi, and personal fees from Amgen and MSD, outside the submitted work.

\section{Funding sources}

The study received no funding. None of the authors have received any payment or incentive to complete the survey.

\section{Introduction}

In a world with an ageing population and inadequate primary prevention strategies for UV protection, the incidence of melanoma and non-melanoma skin cancer is increasing. This has placed a significant burden on societies and presented challenges for healthcare systems to provide efficient care for skin 
cancer patients and their families. ${ }^{1-6}$ In cooperation with several professional organizations, including the European Association for Dermato-Oncology, the European Society of Medical Oncology and the European Society of Surgical Oncology, ${ }^{7}$ the European Cancer Organisation (ECCO) recently published the essential requirements for quality melanoma care. They include the establishment of cancer care pathways that cover the entire patient journey. Also indicated are treatment by multidisciplinary teams in dedicated melanoma centres with patient-centred approaches, audits and quality assurance assessments of outcomes, the education of healthcare professionals and the availability of a high-quality cancer registration system. ${ }^{7}$ Melanoma care pathways, similar to those described by ECCO, have been developed in the United Kingdom (UK) and Australia. ${ }^{8,9}$

The data on the organization of melanoma care in Europe were obtained from previous studies on melanoma and skin cancer care. In 2012, Trakatelli et al. analysed the patient care pathways in 10 countries. Their focus was dermatologist availability, time to dermatologic consultation and follow-up. ${ }^{10}$ A 2013 European Dermatology Health Care Survey evaluated the dermatology workforce and health care in 33 European countries. ${ }^{11}$ The Eurodermoscopy study of the International Dermoscopy Society provided a comprehensive analysis of the availability and clinical use of dermatoscopy in 32 European countries. ${ }^{12,13} \mathrm{~A}$ recent study analysed the global oncology workforce. ${ }^{14}$ These studies found a relationship between the provision of skin cancer care and disease outcomes. ${ }^{5,6}$ Furthermore, a recent article documented a lack of access to medicines for metastatic melanoma that could exacerbate the survival disparities. ${ }^{15}$

The present study gathered comprehensive data on skin cancer diagnosis and treatment practices in Europe to assess compliance with the essential requirements and to highlight the barriers to improving melanoma care.

\section{Materials and methods}

A web-based survey of 32 experts from melanoma centres (23 dermato-oncologists [i.e. dermatologists (DER) with a specialty in oncology], 8 medical oncologists (ONC) and 1 oncological surgeon) in 27 European countries was conducted from 1 February to 1 August 2019. The participants were identified through their publications and leadership positions in national and European scientific organizations. A small proportion (15-25\%) of the data were retrieved from the available national databases and scientific organizations, and a majority (75-85\%) were estimations from current practice. The survey questionnaire collected data on melanoma care pathways (Table S1). This was supplemented with and examined against the physician workforce data from other sources (number of general practitioners (GPs) and surgeons (SURG), 2016 European Commission report; number of DER, DermaSurvey; and number of oncologists, the American Society of Clinical Oncology survey). ${ }^{11,14,16,17-19}$ The data were further correlated with gross national income (GNI) per capita, health expenditure per capita (HEPC), universal health coverage service (UHC; retrieved from the World Bank 2018 database) and mortality-to-incidence ratio (MIR) as a surrogate for survival for 2018. ${ }^{17-19}$ The estimated European standard mortality and incidence rates for 2018 were retrieved from the European Cancer Information System. ${ }^{18}$ The countries were classified as Northern, Western, Southern and Eastern Europe on the basis of the United Nations geoscheme.

The correlations between the variables were estimated with Spearman's, point-biserial or rank-biserial correlation coefficients. The correlations between the dependent variables and the potential predictors were analysed with univariate linear regression in SPSS software. A $P$-value less than 0.05 was considered significant. Multivariate analysis was not performed because of the unfavourable ratio of potential predictors to outcomes.

\section{Results}

\section{Medical specialties and diagnostic techniques regarding melanoma detection}

The survey respondents indicated that the detection of melanoma and skin cancer was done mainly by DER in 18 (67\%) European countries (Table 1). In three countries (Denmark, UK and Hungary [HUN]), GPs also played a significant role. In Belarus (BLR), mainly oncologists were involved in skin cancer detection. In Montenegro and Poland (POL), SURG were mainly involved (Table 1).

The respondents in every country indicated that dermatoscopy was used for melanoma and skin cancer detection. However, the percentage of DER using this technique varied from 10\% in BLR to nearly $100 \%$ in Germany (DEU), the Netherlands and Spain. There was a statistical difference in the use of dermatoscopy in Northern and Western Europe (NWE) and Southern and Eastern Europe (SEE; $98 \%$ vs. $77 \%, P<0.05$ ).

According to the respondents, computerized digital dermatoscopy (CDD) was available in $20(74 \%)$ countries. In NWE, CDD was available in university centres $(60 \%)$ and private practice settings (25\%). In SEE, it was used mainly in private practice $(48 \%)$ and less commonly in university centres (32\%). Reflectance confocal microscopy (RCM) was available in 15 countries (7/11 in NWE and 8/15 in SEE, $P>0.05)$, and optical coherence tomography (OCT) was available in 5 countries in university centres only.

\section{Melanoma surgery and histopathology}

According to the respondents, excisions with primary closure and excisions with skin flaps for melanoma and skin cancer were performed by DER, plastic, ENT/maxillofacial and oncological surgeons (OS) in 25 (92.5\%) and 17 (63\%) countries, respectively (Table 2). Surgical procedures with skin grafts were performed by plastic and ENT/maxillofacial surgeons (MFS) in every country and also by DER in 13 (48\%) countries. 
Table 1 Medical specialties involved and diagnostic techniques used in clinical diagnosis of melanoma in Europe

\begin{tabular}{|c|c|c|c|c|c|c|c|c|}
\hline \multirow[t]{2}{*}{ Country } & \multicolumn{4}{|c|}{ Medical specialty involved (\%)* } & \multirow{2}{*}{$\begin{array}{l}\text { Dermatologist using } \\
\text { dermatoscopy }(\%)\end{array}$} & \multicolumn{3}{|c|}{ Availability of diagnostic techniques } \\
\hline & DER & GP & SURG & ONC & & CDD & $\mathrm{RCM}$ & ОСт \\
\hline \multicolumn{9}{|l|}{ Northern Europe } \\
\hline Denmark & 45 & 45 & 5 & 5 & 92 & No & No & No \\
\hline Estonia & 70 & 10 & 20 & 0 & - & No & No & No \\
\hline Latvia & 75 & 10 & 1 & 10 & 80 & Yes & Yes & No \\
\hline Lithuania & 60 & 0 & 30 & 10 & 51 & Yes & No & No \\
\hline Sweden & 80 & 20 & 0 & 0 & 100 & No & No & No \\
\hline UK & 50 & 30 & 15 & 5 & 99 & Yes & Yes & Yes \\
\hline \multicolumn{9}{|l|}{ Western Europe } \\
\hline Austria & 80 & 15 & 5 & 0 & 97 & Yes & Yes & No \\
\hline Belgium & 100 & 0 & 0 & 0 & 90 & Yes & Yes & No \\
\hline France & 90 & 5 & 5 & 0 & 65 & Yes & Yes & No \\
\hline Germany & 80 & 20 & 0 & 0 & 100 & Yes & Yes & Yes \\
\hline The Netherlands & 70 & 30 & 0 & 0 & 100 & Yes & Yes & Yes \\
\hline Switzerland & 70 & 20 & 10 & 0 & 99 & No & No & No \\
\hline \multicolumn{9}{|l|}{ Southern Europe } \\
\hline Albania & 50 & 10 & 30 & 10 & 96 & Yes & Yes & Yes \\
\hline Bosnia and Herzegovina & 98 & 2 & 0 & 0 & 60 & No & No & No \\
\hline Croatia & 80 & 10 & 5 & 2 & 69 & Yes & No & No \\
\hline Greece & 70 & 5 & 20 & 5 & 75 & Yes & Yes & No \\
\hline Italy & 70 & 5 & 10 & 5 & 80 & Yes & Yes & Yes \\
\hline Montenegro & 30 & 10 & 40 & 20 & 25 & No & No & No \\
\hline Portugal & 80 & 15 & 4 & 1 & 90 & Yes & Yes & No \\
\hline Serbia & 65 & 5 & 20 & 10 & 70 & No & No & No \\
\hline Slovenia & 80 & 20 & 0 & 0 & 90 & Yes & No & No \\
\hline Spain & 100 & & 0 & 0 & 80 & Yes & Yes & No \\
\hline \multicolumn{9}{|l|}{ Eastern Europe } \\
\hline Belarus & 5 & 5 & 5 & 80 & 10 & Yes & No & No \\
\hline Czech Republic & 90 & 2 & 6 & 2 & 80 & Yes & No & No \\
\hline Hungary & 60 & 25 & 10 & 5 & 90 & Yes & Yes & No \\
\hline Poland & 50 & 5 & 5 & 10 & 60 & Yes & Yes & No \\
\hline Romania & 80 & 5 & 10 & 0 & - & Yes & Yes & No \\
\hline Median/total (\%) & 73 & 10 & 10 & 5 & 80 & $20(74 \%)$ & $15(55.5 \%)$ & $5(18.5 \%)$ \\
\hline
\end{tabular}

CDD, computerised digital dermoscopy; DER, dermatologists; GP, general practitioners; OCT, optical coherence tomography; ONC, medical oncologists; RCM, reflectance confocal microscopy; SURG, surgeons.

*Estimated involvement of different medical specialties in clinical diagnosis of skin cancer, i.e. estimated percentage of patients that are coming for skin examination with a suspicion for skin cancer.

Microscopically controlled surgery (Mohs micrographic surgery, surgery with $3 \mathrm{D}$ histology) was unavailable or not used in melanoma treatment in $9(33 \%)$ countries. According to the respondents, this procedure was performed by DER in $13(48 \%)$ countries, plastic surgeons (PS) in $12(44 \%)$ countries, MFS in 3 (11\%) countries and OS in POL. Sentinel lymph node biopsy (SLNB) was performed by OS in $20(74 \%)$ countries, PS in 16 (59\%), MFS in $12(44 \%)$ and DER in $6(22 \%)$ countries. In POL and BLR, there were medical specialty-related restrictions on skin cancer surgery.

Histopathology for skin cancer diagnosis was performed by dermatopathologists and pathologists (P) in 9 (33\%) countries.
In $17(63 \%)$ countries, DER were not involved in the histopathological diagnosis of skin cancer because only $\mathrm{P}$ were allowed to sign histopathological reports.

\section{Systemic treatment of metastatic melanoma}

Metastatic melanoma patients were treated by ONC in all European countries and by dermato-oncologists in 11 (40\%). The systemic treatment of stage III and IV melanoma was administered (for $\geq 70 \%$ patients) mainly by ONC in 21 countries and by dermato-oncologists in Austria, France, DEU, the Czech Republic and HUN (Table 3). Intralesional treatment was administered by dermato-oncologists in 13 countries, 
Table 2 Melanoma surgery in Europe

\begin{tabular}{|c|c|c|c|c|c|c|}
\hline \multirow[t]{2}{*}{ Country } & \multicolumn{5}{|l|}{ Type of surgery } & \multirow{2}{*}{$\begin{array}{l}\text { SLNB } \\
\text { availability } \\
\text { (\%) }\end{array}$} \\
\hline & $\begin{array}{l}\text { Surgery with primary } \\
\text { closure }\end{array}$ & $\begin{array}{l}\text { Surgery with } \\
\text { skin flaps }\end{array}$ & $\begin{array}{l}\text { Surgery with } \\
\text { skin grafts }\end{array}$ & $\begin{array}{l}\text { Microscope. } \\
\text { controlled } \\
\text { surgery }\end{array}$ & SLNB & \\
\hline \multicolumn{7}{|l|}{ Northern Europe } \\
\hline Denmark & $\mathrm{D}, \mathrm{PS}, \mathrm{GS}$ & PS & PS & Not used & PS & 98 \\
\hline Estonia & D, PS, MFS/ENT, OS & $\begin{array}{l}\text { PS, MFS/ENT, } \\
\text { GS, OS }\end{array}$ & MFS/ENT, OS, PS & Not used & MFS/ENT, OS & 95 \\
\hline Latvia & D, PS, MFS/ENT, OS, GS & D, MFS/ENT & PS, MFS & Not available & os & 75 \\
\hline Lithuania & D, PS, MFS/ENT, OS & $\begin{array}{l}\text { PS, MFS/ENT, } \\
\text { OS, GS }\end{array}$ & PS, OS, MFS/ENT & PS & PS, OS & 60 \\
\hline Sweden & D, PS, MFS/ENT, GS & $\begin{array}{l}\text { D, PS, MFS/ENT, } \\
\text { GS }\end{array}$ & D, PS, MFS/ENT & $\mathrm{D}$ & MFS/ENT, GS & 90 \\
\hline UK & D, PS, MFS/ENT, GS & D, PS, MFS/ENT & D, PS, MFS/ENT, OS & D, PS & PS & 80 \\
\hline \multicolumn{7}{|l|}{ Western Europe } \\
\hline Austria & D, PS, GS & $\mathrm{D}, \mathrm{PS}, \mathrm{GS}$ & $\mathrm{D}, \mathrm{PS}, \mathrm{GS}$ & $\mathrm{D}, \mathrm{PS}$ & $\mathrm{D}, \mathrm{PS}$ & 95 \\
\hline Belgium & $\mathrm{D}, \mathrm{PS}, \mathrm{MFS} / \mathrm{ENT}$, OS & D, PS, MFS/ENT & $\mathrm{D}, \mathrm{PS}, \mathrm{MFS} / \mathrm{ENT}$ & $\mathrm{D}, \mathrm{PS}$ & OS & 85 \\
\hline France & $\mathrm{D}, \mathrm{PS}, \mathrm{MFS} / \mathrm{ENT}, \mathrm{GS}$ & $\mathrm{D}, \mathrm{PS}, \mathrm{MFS} / \mathrm{ENT}$ & PS, MFS/ENT, OS & PS, MFS/ENT, OS & PS, MFS/ENT, OS & 90 \\
\hline Germany & $\mathrm{D}, \mathrm{PS}, \mathrm{MFS} / \mathrm{ENT}$, OS & $\begin{array}{l}\text { D, PS, MFS/ENT, } \\
\text { GS }\end{array}$ & D, PS, MFS/ENT, GS & $\mathrm{D}, \mathrm{PS}, \mathrm{MFS} / \mathrm{ENT}$ & D, MFS/ENT & 90 \\
\hline The Netherlands & D, PS, MFS/ENT & D, PS, MFS/ENT & $\begin{array}{l}\text { D, PS, MFS/ENT, } \\
\text { OS, GS }\end{array}$ & $\mathrm{D}$ & MFS/ENT, OS & 90 \\
\hline Switzerland & $\mathrm{D}, \mathrm{PS}, \mathrm{MFS} / \mathrm{ENT}$, GS & $\mathrm{D}, \mathrm{PS}, \mathrm{MFS} / \mathrm{ENT}$ & D, PS, MFS/ENT & $\mathrm{D}, \mathrm{PS}$ & PS, MFS/ENT & 80 \\
\hline \multicolumn{7}{|l|}{ Southern Europe } \\
\hline Albania & D, PS, MFS/ENT, OS, GS & PS, OS, GS & PS, OS & PS & OS & 20 \\
\hline $\begin{array}{l}\text { Bosnia and } \\
\text { Herzegovina }\end{array}$ & D, PS, MFS/ENT, OS, GS & $\begin{array}{l}\text { PS, MFS/ENT, } \\
\text { OS, GS }\end{array}$ & PS, MFS/ENT & Not available & PS & 95 \\
\hline Croatia & D, PS, MFS/ENT, OS, GS & $\begin{array}{l}\text { D, PS, MFS/ENT, } \\
\text { OS, GS }\end{array}$ & PS, MFS/ENT, OS & Not available & PS, MFS/ENT, OS & 95 \\
\hline Greece & D, PS, MFS/ENT, OS, GS & $\mathrm{D}, \mathrm{PS}, \mathrm{OS}, \mathrm{GS}$ & D, PS, OS & Not available & PS, OS & 95 \\
\hline Italy & D, PS, MFS/ENT, OS, GS & $\begin{array}{l}\text { D, PS, MFS/ENT, } \\
\text { OS, GS }\end{array}$ & PS, MFS/ENT, OS, GS & D, PS, MFS/ENT & $\begin{array}{l}\text { D, PS, MFS/ENT, } \\
\text { OS, GS }\end{array}$ & 50 \\
\hline Montenegro & PS, GS & PS & PS & Not available & PS & 90 \\
\hline Portugal & D, PS, MFS/ENT, OS, GS & $\begin{array}{l}\text { D, PS, MFS/ENT, } \\
\text { OS, GS }\end{array}$ & D, PS, MFS/ENT, OS & $\mathrm{D}$ & D, PS, OS & 90 \\
\hline Serbia & D, PS, MFS/ENT, OS, GS & $\begin{array}{l}\text { PS, MFS/ENT, } \\
\text { OS }\end{array}$ & PS, MFS/ENT, OS & $\mathrm{D} \dagger$ & PS, MFS/ENT, OS & 40 \\
\hline Slovenia & D, PS, MFS/ENT, OS, GS & $\begin{array}{l}\text { PS, MFS/ENT, } \\
\text { OS, GS }\end{array}$ & PS, MFS/ENT, OS & Not available & os & 95 \\
\hline Spain & D, PS, MFS/ENT, GS & D, PS & D, PS & D & D, PS, MFS/ENT, GS & 95 \\
\hline \multicolumn{7}{|l|}{ Eastern Europe } \\
\hline Belarus & MFS/ENT, OS, GS & $\begin{array}{l}\text { PS, MFS/ENT, } \\
\text { OS }\end{array}$ & PS, MFS/ENT, OS & Not available & OS & 20 \\
\hline Czech Republic & D, PS, MFS/ENT, GS & $\begin{array}{l}\text { D, PS, MFS/ENT, } \\
\text { GS }\end{array}$ & D, PS, MFS/ENT & $\mathrm{D}$ & $\begin{array}{l}\text { D, PS, MFS/ENT, } \\
\text { OS, GS }\end{array}$ & 95 \\
\hline Hungary & D, PS, MFS/ENT, OS, GS & D, PS, MFS/ENT & D, PS, MFS/ENT & PS & $\begin{array}{l}\text { PS, MFS/ENT, } \\
\text { OS, GS }\end{array}$ & 70 \\
\hline Poland & D, PS, OS, GS & PS, OS & PS, OS & PS, OS & os & 90 \\
\hline Romania & D, PS, MFS/ENT, OS, GS & $\begin{array}{l}\text { D, PS, MFS/ENT, } \\
\text { OS, GS }\end{array}$ & $\begin{array}{l}\text { D, PS, OS, GS, } \\
\text { MFS/ENT }\end{array}$ & $\mathrm{D} \uparrow, \mathrm{PS} \dagger$ & OS & 30 \\
\hline
\end{tabular}

D, dermatologists; GS, general surgeons; MFS/ENT, maxillofacial and/or ear, nose and throat surgeons; OS, oncological surgeons; PS, plastic surgeons; SLNB, sentinel lymph node biopsy.

*Percentage of patients in whom SLNB is indicated and performed.

$\dagger$ Available only in private practice, not reimbursed. 
Table 3 Medical specialities in systemic treatment of melanoma and non-melanoma skin cancer in Europe

\begin{tabular}{|c|c|c|c|c|c|c|c|}
\hline \multirow[t]{2}{*}{ Country } & \multicolumn{2}{|l|}{ Adjuvant* } & \multicolumn{3}{|l|}{ Intralesional* } & \multicolumn{2}{|c|}{$\begin{array}{l}\text { Systemic for unresectable } \\
\text { stage III and stage IV } \\
\text { melanoma* }\end{array}$} \\
\hline & $\begin{array}{l}\text { Medical } \\
\text { oncologists }\end{array}$ & $\begin{array}{l}\text { Dermato- } \\
\text { oncologist }\end{array}$ & $\begin{array}{l}\text { Medical } \\
\text { oncologists }\end{array}$ & $\begin{array}{l}\text { Dermato- } \\
\text { oncologist }\end{array}$ & $\begin{array}{l}\text { Surgical } \\
\text { oncologist }\end{array}$ & $\begin{array}{l}\text { Medical } \\
\text { oncologists }\end{array}$ & $\begin{array}{l}\text { Dermato- } \\
\text { oncologist }\end{array}$ \\
\hline \multicolumn{8}{|l|}{ Northern Europe } \\
\hline Denmark & 100 & 0 & 100 & 0 & 0 & 100 & 0 \\
\hline Estonia & 100 & 0 & 0 & 0 & 0 & 100 & 0 \\
\hline Lithuania & 100 & 0 & 0 & 0 & 0 & 100 & 0 \\
\hline Latvia & 100 & 0 & 0 & 0 & 0 & 95 & 5 \\
\hline Sweden & 100 & 0 & 50 & 0 & 50 & 100 & 0 \\
\hline UK & 100 & 0 & 100 & 0 & 0 & 100 & 0 \\
\hline \multicolumn{8}{|l|}{ Western Europe } \\
\hline Austria & 5 & 95 & 0 & 100 & 0 & 5 & 95 \\
\hline Belgium & 100 & 0 & 100 & 0 & 0 & 100 & 0 \\
\hline France & 10 & 90 & 0 & 90 & 10 & 10 & 90 \\
\hline Germany & 20 & 80 & 0 & 95 & 5 & 20 & 80 \\
\hline The Netherlands & 100 & 0 & 0 & & 0 & 100 & 0 \\
\hline Switzerland & 50 & 50 & 20 & 80 & 0 & 60 & 40 \\
\hline \multicolumn{8}{|l|}{ Southern Europe } \\
\hline Albania & 100 & 0 & 10 & 60 & 30 & 100 & 0 \\
\hline Bosnia and Herzegovina & 100 & 0 & 0 & 100 & 0 & 100 & 0 \\
\hline Croatia & 100 & 0 & 0 & & 0 & 100 & 0 \\
\hline Greece & 100 & 0 & 70 & 30 & 0 & 100 & 0 \\
\hline Italy & 70 & 30 & 100 & 0 & 0 & 50 & 50 \\
\hline Montenegro & 100 & 0 & 0 & 0 & 0 & 100 & 0 \\
\hline Portugal & 95 & 5 & 50 & 50 & 0 & 95 & 5 \\
\hline Serbia & 70 & 25 & 0 & 15 & 85 & 75 & 25 \\
\hline Slovenia & 100 & 0 & 100 & 0 & 0 & 100 & 0 \\
\hline Spain & 80 & 20 & 0 & 100 & 0 & 80 & 20 \\
\hline \multicolumn{8}{|l|}{ Eastern Europe } \\
\hline Belarus & 100 & 0 & 0 & 0 & 100 & 100 & 0 \\
\hline Czech Republic & 20 & 80 & 50 & 50 & 0 & 30 & 70 \\
\hline Hungary & 20 & 80 & 0 & 100 & 0 & 20 & 80 \\
\hline Poland & 100 & 0 & 100 & 0 & 0 & 100 & 0 \\
\hline Romania & 100 & 0 & 5 & 80 & 10 & 100 & 0 \\
\hline
\end{tabular}

*Estimated percentage of patients treated by different medical disciplines.

ONC in 13 and surgical oncologists in 7 countries. In 15 countries, there were legislative and/or reimbursement restrictions on the prescription of systemic melanoma treatment by specialists other than ONC. Clinical trials were performed by specialists who were already involved in the systemic treatment of melanoma.

\section{Organization of melanoma care}

In this survey, melanoma care units (i.e. pigment lesion clinics, urgent access melanoma specialty care clinics) were defined as clinics in which patients with suspected melanoma had fast access to and priority status for surgical treatment and diagnostic work-up on the basis of primary care (GPs, primary care DER). It was estimated that quick access upon primary care referrals was available in $19(70 \%)$ countries: all the countries in NWE and 8 (53\%) in SEE (Table 4).

The follow-up for low-risk melanoma was organized mainly in general hospitals in 15 countries, in tertiary and comprehensive cancer centres in 10 countries and mainly in private practice settings in 2 countries (Table 5). Dermatologists were primarily responsible for the follow-up of low-risk melanoma $(83 \%$ of countries of NWE, $67 \%$ of countries in SEE). For localized highrisk melanoma, DER were responsible for follow-up in 9/12 (75\%) of NWE countries, and 7/15 (47\%) countries of SEE. Medical oncologists and SURG were also involved in follow-up in 5/15 (33\%) and 3/15 (20\%) countries, respectively. In Portugal, GPs were reportedly responsible for follow-up of all localized melanoma cases. Patients with stage III melanoma were 
Table 4 Organization of melanoma care in Europe

\begin{tabular}{|c|c|c|c|c|c|c|}
\hline Country & $\begin{array}{l}\text { Melanoma } \\
\text { care units }\end{array}$ & $\begin{array}{l}\text { Accreditation of } \\
\text { oncology centres }\end{array}$ & $\begin{array}{l}\text { Quality control } \\
\text { process of } \\
\text { oncology centres }\end{array}$ & $\begin{array}{l}\text { Multidisciplinary } \\
\text { tumour board }\end{array}$ & $\begin{array}{l}\text { Core specialties } \\
\text { in MTB }\end{array}$ & $\begin{array}{l}\text { Molecular } \\
\text { oncology } \\
\text { tumour board }\end{array}$ \\
\hline \multicolumn{7}{|l|}{ Northern Europe } \\
\hline Denmark & Yes & No & No & Yes & $\mathrm{MO} \dagger, \mathrm{PS}, \mathrm{P}, \mathrm{R}, \mathrm{RT}$ & No \\
\hline Estonia & Yes & Yes & No & Yes & $\mathrm{D}, \mathrm{MO}+, \mathrm{OS}, \mathrm{P}, \mathrm{R}, \mathrm{RT}$ & Yes \\
\hline Latvia & Yes & No & No & No & $\mathrm{MO} \dagger, \mathrm{OS} \uparrow$ & No \\
\hline Lithuania & No & No & No & Yes & $\mathrm{D}, \mathrm{MO} \dagger, \mathrm{OS}, \mathrm{PS}, \mathrm{P}, \mathrm{R}, \mathrm{RT}$ & No \\
\hline Sweden & Yes & No & No & Yes & $\mathrm{D} \dagger, \mathrm{MO} \dagger, \mathrm{OS} \uparrow, \mathrm{PS}, \mathrm{P}, \mathrm{R}, \mathrm{RT}$ & No \\
\hline UK & Yes & Yes & Yes & Yes & $\mathrm{D}, \mathrm{MO}, \mathrm{OS}, \mathrm{PS}, \mathrm{P}, \mathrm{R}, \mathrm{RT}$ & \\
\hline \multicolumn{7}{|l|}{ Western Europe } \\
\hline Austria & Yes & Yes & Yes & Yes & $\mathrm{D} \dagger, \mathrm{MO}, \mathrm{OS}, \mathrm{PS}, \mathrm{P}, \mathrm{R}, \mathrm{RT}$ & No \\
\hline Belgium & Yes & Yes & Yes & Yes & $\mathrm{D} \uparrow, \mathrm{MO}, \mathrm{OS}, \mathrm{PS}, \mathrm{P}, \mathrm{R}, \mathrm{RT}$ & No \\
\hline France & Yes & Yes & Yes & Yes & $\mathrm{D} \dagger, \mathrm{MO}, \mathrm{OS}, \mathrm{PS}, \mathrm{P}, \mathrm{R}, \mathrm{RT}$ & Yes \\
\hline Germany & Yes & Yes & Yes & Yes & $\mathrm{D \dagger}, \mathrm{MO}, \mathrm{OS}, \mathrm{PS}, \mathrm{R}, \mathrm{RT}$ & Yes \\
\hline The Netherlands & Yes & Yes & Yes & Yes & $\mathrm{D}^{\dagger}, \mathrm{MO}^{\dagger}, \mathrm{OS}, \mathrm{PS}, \mathrm{R}, \mathrm{RT}, \mathrm{P}$ & No \\
\hline Switzerland & Yes & Yes & Yes & Yes & $\mathrm{D} \dagger, \mathrm{MO}, \mathrm{PS}, \mathrm{P}, \mathrm{R}, \mathrm{RT}$ & Yes \\
\hline \multicolumn{7}{|l|}{ Southern Europe } \\
\hline Albania & No & No & No & Yes & $\mathrm{D}, \mathrm{MO} \dagger, \mathrm{OS}, \mathrm{PS}, \mathrm{P}, \mathrm{R}, \mathrm{RT}$ & No \\
\hline Bosnia and Herzegovina & No & No & No & Yes & $\mathrm{D}, \mathrm{MO} \dagger, \mathrm{PS}, \mathrm{P}, \mathrm{RT}$ & No \\
\hline Croatia & Yes & Yes & Yes & Yes & $\mathrm{D} \uparrow, \mathrm{MO} \dagger, \mathrm{OS}, \mathrm{PS}, \mathrm{P}, \mathrm{R}, \mathrm{RT}$ & No \\
\hline Greece & No & No & No & Yes & $\mathrm{D}, \mathrm{MO}+, \mathrm{OS}, \mathrm{PS}, \mathrm{RT}$ & No \\
\hline Italy & Yes & Yes & Yes & Yes & $\mathrm{D}, \mathrm{MO} \dagger, \mathrm{OS}, \mathrm{PS}, \mathrm{P}, \mathrm{R}, \mathrm{RT}$ & Yes \\
\hline Montenegro & Yes & No & Yes & Yes & $\mathrm{D}, \mathrm{MO}, \mathrm{PS} \dagger, \mathrm{RT}$ & No \\
\hline Portugal & No & Yes & No & Yes & $\mathrm{D} \dagger, \mathrm{MO}, \mathrm{OS}, \mathrm{PS}, \mathrm{P}, \mathrm{R}, \mathrm{RT}$ & Yes \\
\hline Serbia & No & No & No & Yes & $\mathrm{D} \dagger, \mathrm{MO} \dagger, \mathrm{OS} \dagger, \mathrm{PS}, \mathrm{P}, \mathrm{R}, \mathrm{RT}$ & No \\
\hline Slovenia & No & Yes & Yes & Yes & $\mathrm{D}, \mathrm{MO} \dagger, \mathrm{OS} \uparrow, \mathrm{P}, \mathrm{R}, \mathrm{RT}$ & Yes \\
\hline Spain & Yes & No & No & Yes & $\mathrm{D} \dagger, \mathrm{MO} \dagger, \mathrm{PS}, \mathrm{P}, \mathrm{R}, \mathrm{RT}$ & No \\
\hline \multicolumn{7}{|l|}{ Eastern Europe } \\
\hline Belarus & Yes & Yes & Yes & Yes & $\mathrm{MO} \dagger, \mathrm{OS} \uparrow, \mathrm{P}, \mathrm{R}, \mathrm{RT}$ & Yes \\
\hline Czech Republic & Yes & No & No & Yes & $\mathrm{D} \dagger, \mathrm{MO}, \mathrm{OS}, \mathrm{PS}, \mathrm{P}, \mathrm{RT}$ & No \\
\hline Hungary & Yes & Yes & Yes & Yes & $\mathrm{D} \dagger, \mathrm{MO}, \mathrm{OS}, \mathrm{PS}, \mathrm{P}, \mathrm{R}, \mathrm{RT}$ & Yes \\
\hline Poland & Yes & Yes & No & Yes & $\mathrm{MO}, \mathrm{OS} \uparrow, \mathrm{P}, \mathrm{R}, \mathrm{RT}$ & Yes \\
\hline Romania & No & Yes & Yes & Yes & $\mathrm{D}, \mathrm{MO} \dagger, \mathrm{OS}, \mathrm{P}, \mathrm{R}$ & No \\
\hline
\end{tabular}

$\mathrm{MO}$, medical oncologists; OS, oncologic surgeon; P, pathologist; PS, plastic surgeon; R, radiologist; RT, radiotherapist.

*Melanoma care units - clinics in which patients with suspected melanoma had fast access to surgical treatment and diagnostic work-up on the basis of primary care referral (i.e. pigment lesion clinic).

†Organisation of multidisciplinary tumor board (MTB).

referred mainly to tertiary or comprehensive cancer centres. Oncologists were responsible for follow-up in 13 countries, DER in 10 and SURG in 6 countries.

Quality assurance, auditing and the accreditation of oncology centres are also essential to the establishment and maintenance of high-quality cancer care. The process of accreditation differs between countries. Any type of accreditation process for oncology centres was found to be established in 16 countries (67\% in NWE, $53 \%$ in SEE). Fourteen of these countries had quality control processes ( $58 \%$ in NWE, $47 \%$ in SEE). MTBs were organized by ONC in 11 countries, DER in 8 and by both in 5 countries, while SURG were also involved in 6 countries (Table 4).
The survey data indicated that melanoma patients had access to multidisciplinary tumour boards (MTBs) in 23/27 (85\%) countries and the following core medical specialties: ONC (27countries), SURG (27 countries), DER (23 countries), radiotherapists (24 countries), P (22 countries) and radiologists (22 countries). Molecular oncology tumour boards were available in 10 countries (4/12 in NWE and 6/15 in SEE; Table 4).

\section{Melanoma registries in Europe}

Table 6 presents the availability of melanoma registries in Europe. National population cancer registries were established in $17 / 24(71 \%)$ countries ( $90 \%$ in NWE, $50 \%$ in SEE), and 
Table 5 Follow-up of melanoma in Europe

\begin{tabular}{|c|c|c|c|c|c|c|}
\hline \multirow[t]{3}{*}{ Country } & \multicolumn{6}{|c|}{ Melanoma stage } \\
\hline & \multicolumn{2}{|c|}{ Low-risk localized (IA) } & \multicolumn{2}{|c|}{$\begin{array}{l}\text { Intermediate and } \\
\text { localized (IB-IIC) }\end{array}$} & \multicolumn{2}{|c|}{ Metastatic stage III } \\
\hline & $\begin{array}{l}\text { Medical } \\
\text { specialty* }\end{array}$ & Institution $\dagger$ & $\begin{array}{l}\text { Medical } \\
\text { specialty* }\end{array}$ & Institution $\dagger$ & $\begin{array}{l}\text { Medical } \\
\text { specialty* }\end{array}$ & Institution $\dagger$ \\
\hline \multicolumn{7}{|l|}{ Northern Europe } \\
\hline Denmark & GP & SGH & $S$ & TOC & $S$ & TOC \\
\hline Estonia & $\mathrm{D}$ & $\mathrm{CCC}$ & $\mathrm{D}$ & $\mathrm{CCC}$ & $\mathrm{MO}$ & $\mathrm{CCC}$ \\
\hline Latvia & GP & TOC & GP & TOC & $\mathrm{MO}$ & TOC \\
\hline Lithuania & $\mathrm{D}$ & TOC & MO & TOC & MO & TOC \\
\hline Sweden & $\mathrm{D}$ & $\mathrm{DGH}$ & $\mathrm{D}$ & TOC & S & TOC \\
\hline UK & $\mathrm{D}$ & TOC & D & $\mathrm{CCC}$ & MO & $\mathrm{CCC}$ \\
\hline \multicolumn{7}{|l|}{ Western Europe } \\
\hline Austria & $\mathrm{D}$ & $\mathrm{DGH}$ & $\mathrm{D}$ & $\mathrm{GH}$ & $\mathrm{D}$ & $\mathrm{GH}$ \\
\hline Belgium & $\mathrm{D}$ & SGH & $\mathrm{D}$ & TOC & MO & TOC \\
\hline France & $\mathrm{D}$ & PP & $\mathrm{D}$ & TOC & $\mathrm{D}$ & TOC \\
\hline Germany & $\mathrm{D}$ & DGH & $\mathrm{D}$ & $\mathrm{CCC}$ & $\mathrm{D}$ & $\mathrm{CCC}$ \\
\hline The Netherlands & $\mathrm{D}$ & $\mathrm{DGH}$ & $\mathrm{D}$ & $\mathrm{DGH}$ & $\mathrm{D}, \mathrm{MO}, \mathrm{S}$ & TOC \\
\hline Switzerland & $\mathrm{D}$ & $\mathrm{PP}$ & $D$ & $\mathrm{CCC}$ & $\mathrm{D}$ & $\mathrm{CCC}$ \\
\hline \multicolumn{7}{|l|}{ Southern Europe } \\
\hline Albania & S & $\mathrm{DGH}$ & MO & $\mathrm{CCC}$ & $\mathrm{MO}$ & $\mathrm{CCC}$ \\
\hline Bosnia and Herzegovina & $\mathrm{D}$ & TOC & MO & TOC & $\mathrm{MO}$ & TOC \\
\hline Croatia & $\mathrm{D}$ & $\mathrm{DGH}$ & $\mathrm{D}$ & $\mathrm{CCC}$ & MO & $\mathrm{CCC}$ \\
\hline Greece & $\mathrm{D}$ & $\begin{array}{l}\text { Dermatology } \\
\text { hospitals }\end{array}$ & MO & TOC & MO & TOC \\
\hline Italy & $\mathrm{D}$ & $\mathrm{DGH}$ & $\mathrm{D}$ & $\mathrm{CCC}$ & $\mathrm{D}$ & $\mathrm{CCC}$ \\
\hline Montenegro & $\mathrm{S}$ & $\mathrm{CCC}$ & MO & $\mathrm{CCC}$ & MO & CCC \\
\hline Portugal & GP & DGH & GP & TOC & GP & TOC \\
\hline Slovenia & $\mathrm{S}$ & $\mathrm{CCC}$ & S & $\mathrm{CCC}$ & $\mathrm{S}$ & $\mathrm{CCC}$ \\
\hline Serbia & $\mathrm{D}, \mathrm{S}$ & TOC & $\mathrm{D}, \mathrm{S}$ & TOC & $\mathrm{D}, \mathrm{S}$ & TOC \\
\hline Spain & $\mathrm{D}$ & DGH & D & $\mathrm{GH}$ & $\mathrm{D}$ & $\mathrm{GH}$ \\
\hline \multicolumn{7}{|l|}{ Eastern Europe } \\
\hline Belarus & MO & TOC & MO & $\mathrm{CCC}$ & $\mathrm{MO}$ & $\mathrm{CCC}$ \\
\hline Czech Republic & $\mathrm{D}$ & $\mathrm{DGH}$ & $\mathrm{D}$ & TOC & $D$ & TOC \\
\hline Hungary & $\mathrm{D}$ & $\mathrm{DGH}$ & $D$ & $\mathrm{CCC}$ & $D$ & $\mathrm{CCC}$ \\
\hline Poland & $\mathrm{D}$ & TOC & $S$ & $\mathrm{CCC}$ & $S$ & $\mathrm{CCC}$ \\
\hline Romania & $\mathrm{D}$ & DGH & $\mathrm{D}$ & TOC & $\mathrm{MO}$ & TOC \\
\hline
\end{tabular}

CCC, comprehensive cancer center; D, dermatologists; DGH, dermatological unit in general hospital; GH, general hospital; GP, general practitioners; MO, medical oncologist; PP, private practice; S, surgeons; SGH, surgical units in general hospitals; TOC, tertiary oncology center.

*Medical specialty mainly involved in follow-up of the patients.

$\dagger$ Institution where majority of the patients are referred for follow-up.

melanoma clinical registries were available in 13/24 (54\%) countries (50\% in NWE, 57\% in SEE).

\section{Education}

Skin cancer detection educational programmes for GPs were organized in 17 countries ( $73 \%$ in NWE, 60\% in SEE). Dermatoscopy training was an official aspect of dermatology residency programmes in 20 (74\%) countries (Table 7). However, it was also provided in other countries through dermatoscopy courses and mentorship during residency (Table 7). Dermatosurgery training during dermatology residency was available in 22 countries (92\% in NWE, $73 \%$ in SEE), and dermato-oncology training was available in 22 countries $(75 \%$ in NWE, $87 \%$ in SEE). In 11 countries ( $50 \%$ in NWE, $33 \%$ in SEE), a dermatopathology subspecialty was available to DER and $\mathrm{P}$, and in 2 countries, it was available to $\mathrm{P}$, only. In the 11 countries in which this was not available, DER faced legislative hurdles to perform histopathological analyses of skin cancer. Subspecialty 
Table 6 Melanoma registries in Europe

\begin{tabular}{|c|c|c|c|}
\hline \multirow[t]{2}{*}{ Country } & \multicolumn{3}{|c|}{ Melanoma registry } \\
\hline & $\begin{array}{l}\text { National } \\
\text { cancer } \\
\text { registry }\end{array}$ & $\begin{array}{l}\text { Clinical } \\
\text { registry }\end{array}$ & $\begin{array}{l}\text { Stage of } \\
\text { melanoma } \\
\text { recorded }\end{array}$ \\
\hline \multicolumn{4}{|l|}{ Northern Europe } \\
\hline Denmark & Yes & Yes & Stages I-IV \\
\hline Estonia & Yes & No & Stages I-IV \\
\hline Lithuania & Yes & No & \\
\hline Sweden & Yes & Yes & Stages I-IV \\
\hline UK & Yes & No & \\
\hline \multicolumn{4}{|l|}{ Western Europe } \\
\hline Austria & Yes & No & \\
\hline Belgium & Yes & No & Stages I-IV \\
\hline France & No* & Yes & Stages I-IV \\
\hline Germany & Yes & Yes & Stages I-IV \\
\hline The Netherlands & Yes & Yes & Stages I-IV \\
\hline Switzerland & Yes & Yes & Stages I-IV \\
\hline \multicolumn{4}{|l|}{ Southern Europe } \\
\hline Albania & No & No & \\
\hline Bosnia and Herzegovina & No* & No & \\
\hline Croatia & Yes & Yes & Stages I-IV \\
\hline Greece & No* & No & \\
\hline Italy & No* & No & \\
\hline Montenegro & Yes & No & \\
\hline Portugal & Yes & Yes & Stages I-IV \\
\hline Serbia & No* & Yes & Stages I-IV \\
\hline Slovenia & Yes & Yes & Stages I-IV \\
\hline Spain & Yes & Yes & \\
\hline \multicolumn{4}{|l|}{ Eastern Europe } \\
\hline Belarus & Yes & Yes & Stages I-IV \\
\hline Czech Republic & Yes & Yes & Stages I-IV \\
\hline Poland & Yes & Yes & Stages I-IV \\
\hline Romania & No* & No & \\
\hline
\end{tabular}

*Regional registries exist.

training in dermatosurgery was available in 8 countries (5 in NWE, 3 in SEE), and subspecialty training in oncology was available for DER in 8 countries (33\% in NWE, 27\% in SEE; Table 7).

\section{Correlation of economic and healthcare organization variables with melanoma mortality-to-incidence ratio}

The MIR was calculated from data obtained from the European Cancer Information System. ${ }^{18,20}$ It was correlated with the data on 1-year, 5-year and conditional 5-year survival from the EUROCARE- 5 study of 18 countries for the same year. ${ }^{5}$ A highly significant correlation was found, thus supporting the effectiveness of the MIR as a surrogate marker for survival in the absence of population-based survival data (Table S2). A higher MIR was associated with lower survival rates (Table 8).

The MIR was found to be significantly lower in countries with a higher GNI per capita, HEPC and UHC $(P<0.001)$ and in those with a higher number of GPs and SURG per million inhabitants, higher number of GPs involvement in skin cancer detection and higher percentage of DER using dermatoscopy. In the countries where SURG and oncologists were also involved in the clinical diagnosis of melanoma, the MIR was higher (Fig. 1). The percentage of melanoma patients who underwent SLND (if indicated) was higher in countries with a lower MIR. In the countries where surgical procedures with skin flaps and skin grafts were performed by DER and the histopathology of skin cancer was performed by $\mathrm{P}$ and dermatopathologists, the MIR was significantly lower. The MIR was also lower in countries where DER were involved in the follow-up of stage IB-IIC patients and DER and oncologists were responsible for the follow-up and systemic treatment of stage III and IV patients. In countries where only ONC were responsible for the follow-up of stage III patients, the MIR was significantly higher. The limitations in the prescription of systemic melanoma therapy and restrictions on reimbursement seemed to be correlated with a higher MIR (Table 8). Access to MTBs was significantly associated with a lower MIR, particularly in countries in which they were organized by DER.

Because the economic parameters were significantly correlated with the MIR, further analysis was done to explore the effects of the relationship between HEPC and various aspects of the healthcare system on the melanoma care pathway (Table S3). In countries with a higher HEPC, the GPs and/or DER were educated in the early detection of skin cancer; a higher percentage of DER used dermatoscopy; a higher percentage had a higher level of education in dermatologic surgery, dermatopathology and the systemic treatment of melanoma; and they had a greater involvement in the follow-up and systemic treatment of stage IB-III cancer. In countries with a lower HEPC, oncologists or SURG were responsible for skin cancer detection. In contrast, the workforce (number of DER, P, SURG and oncologists) and the estimated access to CDD and sentinel lymph node dissection were not correlated with the HEPC $(P>0.05)$.

\section{Discussion}

Melanoma was the seventh most frequently diagnosed cancer in the European Union in 2012. The highest incidence and mortality rates were recorded in the Nordic countries, and the lowest, in Southern Europe. ${ }^{1-4,21,22}$ However, when the MIR was used as a proxy for the fatality rate, the highest MIR was in Central and Eastern Europe, and the lowest was in Western Europe. ${ }^{6}$ In addition, the recorded survival rates ranged from $<50 \%$ in Eastern and Southeast Europe to $>90 \%$ in the Nordic countries. ${ }^{5,22}$ Recent data from DEU indicated that the mortality rates were stabilizing, and even decreasing, in the Northwest countries. ${ }^{22,23}$ This was attributed to nationwide screening campaigns and the widespread use of effective systemic treatments for metastatic disease. $^{24}$ In most of Eastern Europe, the first prevention campaigns were introduced in 2008; less effective melanoma care 
Table 7 Education of physicians in skin cancer diagnosis and treatment in Europe

\begin{tabular}{|c|c|c|c|c|c|c|c|}
\hline Country & $\begin{array}{l}\text { Educational } \\
\text { programmes } \\
\text { for GPs }\end{array}$ & $\begin{array}{l}\text { Training in } \\
\text { dermatoscopy as } \\
\text { official part of } \\
\text { dermatology } \\
\text { residency }\end{array}$ & $\begin{array}{l}\text { Training in } \\
\text { dermato-surgery } \\
\text { during dermatology } \\
\text { residency }\end{array}$ & $\begin{array}{l}\text { Training in } \\
\text { dermato-oncology } \\
\text { during } \\
\text { dermatology } \\
\text { residency }\end{array}$ & $\begin{array}{l}\text { Subspecialty } \\
\text { training in } \\
\text { dermato- } \\
\text { pathology }\end{array}$ & $\begin{array}{l}\text { Subspecialty } \\
\text { training in } \\
\text { dermatologic } \\
\text { surgery }\end{array}$ & $\begin{array}{l}\text { Subspecialty } \\
\text { training in } \\
\text { oncology for } \\
\text { dermatologists } \\
\text { (DER) }\end{array}$ \\
\hline \multicolumn{8}{|l|}{ Northern Europe } \\
\hline Denmark & Yes & Yes & & No & No & No & No \\
\hline Estonia & No & No & Yes & Yes & No & No & No \\
\hline Latvia & No & No & No & Yes & No & No & No \\
\hline Lithuania & Yes & Yes & Yes & Yes & No & No & No \\
\hline Sweden & Yes & Yes & Yes & Yes & No & No & No \\
\hline UK & Yes & Yes & Yes & Yes & Yes & Yes & Yes \\
\hline \multicolumn{8}{|l|}{ Western Europe } \\
\hline Austria & Yes & Yes & Yes & Yes & Yes & No & No \\
\hline Belgium & Yes & No & Yes & No & No & Yes & No \\
\hline France & Yes & No & Yes & Yes & Yes & Yes & Yes \\
\hline Germany & Yes & Yes & Yes & Yes & Yes & Yes & Yes \\
\hline The Netherlands & No* & Yes & Yes & Yes & Yes & Yes & Yes \\
\hline Switzerland & Yes & Yes & Yes & Yes & Yes & No & No \\
\hline \multicolumn{8}{|l|}{ Southern Europe } \\
\hline Albania & No & Yes & No & Yes & No & No & No \\
\hline $\begin{array}{l}\text { Bosnia and } \\
\text { Herzegovina }\end{array}$ & No & Yes & No & Yes & Yes & No & Yes \\
\hline Croatia & Yes & Yes & Yes & Yes & No & No & Yes \\
\hline Greece & No & Yes & Yes & Yes & No & No & No \\
\hline Italy & No & Yes & Yes & Yes & Yes & Yes & Yes \\
\hline Montenegro & Yes & No & Yes & Yes & No & No & No \\
\hline Portugal & Yes & Yes & Yes & Yes & Yes & No & No \\
\hline Serbia & Yes & Yes & Yes & Yes & No & No & Yes \\
\hline Slovenia & Yes & Yes & Yes & No & No & No & No \\
\hline Spain & Yes & No & Yes & Yes & No & No & No \\
\hline \multicolumn{8}{|l|}{ Eastern Europe } \\
\hline Belarus & Yes & No & No & Yes & No & No & No \\
\hline Czech Republic & No & Yes & Yes & Yes & Yes & Yes & No \\
\hline Hungary & No & Yes & Yes & Yes & Yes & No & No \\
\hline Poland & Yes & Yes & Yes & Yes & No & No & No \\
\hline Romania & Yes & Yes & Yes & No & No & Yes & No \\
\hline Total (yes/no + yes), \% & $18 / 27,66 \%$ & $20(74 \%)$ & $23(85 \%)$ & $23(85 \%)$ & $11(40 \%)$ & $8(30 \%)$ & $8(30 \%)$ \\
\hline
\end{tabular}

*Percentage of patients in whom SLNB is indicated and performed.

and significant delays in access to effective systemic treatments led to lower survival rates. ${ }^{15,23,25}$

The ECCO recently developed essential requirements for an optimal melanoma care pathway. However, the current survey found significant differences in the extent to which the countries had met these requirements. ${ }^{7}$ In the countries where GPs and DER were educated and actively involved in clinical diagnosis of skin cancer and a higher percentage of DER were using dermatoscopy, the MIR seemed to be lower (Fig. 1, Table 7). On the contrary, the countries in which SURG and oncologists were also involved in clinical diagnosis of skin cancer had the highest MIR. This highlights the need for patients to have broader access to medical professionals who are skilled in the detection of skin cancer, with DER trained in dermatoscopy being the leaders in the field. These results confirm those of recent studies in the United States where a lower MIR was found to be correlated with dermatologist and primary care provider density. ${ }^{26,27}$ The active collaboration of DER and GPs in the early diagnosis of skin cancer is crucial to preventing dermatology offices from being overloaded with unselected patients, thereby making access for patients with skin cancer very difficult. ${ }^{28-30}$ In this regard, education of GPs in skin cancer detection and DER in dermatosurgery and dermatopathology is very important. In the current survey, advanced education in dermatosurgery and 
Table 8 Correlation of economic parameters and various components of melanoma care pathway to melanoma mortality-to-incidence ratio as a surrogate of survival

\begin{tabular}{|c|c|c|c|}
\hline \multirow[t]{2}{*}{ Variable } & \multicolumn{3}{|c|}{$\begin{array}{l}\text { Correlation mortality-to-incidence ratio (MIR) in } \\
\text { European countries* }\end{array}$} \\
\hline & B & $95 \% \mathrm{Cl}$ & $P$-value \\
\hline \multicolumn{4}{|l|}{ Economic variables } \\
\hline Gross national income per capita* & -0.001 & $-0,001$ to $<0,001$ & $<0.001$ \\
\hline Universal healthcare coverage* & -1.295 & -1.728 to -0.862 & $<0.001$ \\
\hline Health expenditure per capita* & -0.005 & -0.006 to -0.004 & $<0.001$ \\
\hline \multicolumn{4}{|l|}{ Physicians workforce (number per 1 million inhabitants) } \\
\hline General practitioners & $<0.001$ & $<0.001$ to $<0.001$ & 0.079 \\
\hline Surgeons & $<0.001$ & $<0.001$ to $<0.001$ & 0.091 \\
\hline Pathologist & -0.004 & -0.024 to 0.017 & 0.703 \\
\hline Dermatologists & -0.182 & -0.405 to 0.042 & 0.105 \\
\hline Oncologists & -0.003 & -0.009 to 0.004 & 0.378 \\
\hline \multicolumn{4}{|l|}{ Medical specialties involved and diagnostic techniques used in clinical diagnosis of melanoma } \\
\hline General practitioners & -0.451 & -0.829 to -0.073 & 0.021 \\
\hline GPs access to educational programmes for skin cancer detection & -3.188 & -12.866 to 6.491 & 0.503 \\
\hline Dermatologists & -0.156 & -0.363 to 0.051 & 0.132 \\
\hline Percentage of DER using dermatoscopy & -0.273 & -0.444 to -0.103 & 0.003 \\
\hline Surgeons & 0.577 & 0.234 to 0.920 & 0.002 \\
\hline Oncologists & 0.187 & -0.085 to 0.46 & 0.169 \\
\hline Access to CDD & 0.987 & -9.003 to 10.977 & 0.840 \\
\hline Access to reflectance confocal microscopy (RCM) & -1.750 & -10.909 to 7.409 & 0.697 \\
\hline \multicolumn{4}{|l|}{ Melanoma surgery } \\
\hline Access to microscopically controlled surgery & 6.296 & -3.36 to 15.952 & 0.191 \\
\hline Access to SLND (\% of patients with performed SLND when indicated) & -0.186 & -0.372 to -0.001 & 0.049 \\
\hline Dermatologists performing surgical procedures with skin flaps & -11.047 & -19.334 to -2.760 & 0.011 \\
\hline Dermatologists performing surgical procedures with skin grafts & -13.729 & -21.305 to -6.154 & 0.001 \\
\hline \multicolumn{4}{|l|}{ Histopathology } \\
\hline Histopathology performed by both dermatopathologists and pathologists $(P)$ & -8.333 & -17.389 to 0.722 & 0.070 \\
\hline \multicolumn{4}{|l|}{ Follow-up of melanoma patients } \\
\hline Dermatologists responsible for follow-up of stage IA_ & -7.767 & -17.785 to 2.251 & 0.123 \\
\hline Dermatologists responsible for follow-up of stage IB-IIC & -11.436 & -19.579 to -3.293 & 0.008 \\
\hline Dermatologists responsible for follow-up of stage stage IIIA & -12.294 & -20.644 to -3.944 & 0.006 \\
\hline Oncologists responsible for follow-up of stage stage IIIA & 11.536 & 3.517 to 19.555 & 0.007 \\
\hline \multicolumn{4}{|l|}{ Systemic treatment for metastatic melanoma } \\
\hline Adjuvant treatment prescribed by both dermato-oncologists and medical oncologists (ONC) & -0.140 & -0.265 to -0.014 & 0.031 \\
\hline Adjuvant treatment prescribed only by ONC & 2.176 & -2.682 to 7.034 & 0.364 \\
\hline Systemic treatment prescribed by both dermato-oncologists and ONC & -0.141 & -0.269 to -0.012 & 0.033 \\
\hline Systemic treatment prescribed only by ONC & 0.145 & 0.013 to 0.276 & 0.032 \\
\hline Legislative restrictions to prescribe systemic melanoma therapy based on medical specialty & 8.533 & -0.146 to 17.213 & 0.054 \\
\hline Limitations to reimbursement of systemic melanoma therapy based on medical specialty & 10.889 & 2.143 to 19.635 & 0.017 \\
\hline \multicolumn{4}{|l|}{ Organization of melanoma care } \\
\hline Melanoma care units with fast access from primary care & -6.921 & -16.941 to 3.098 & 0.167 \\
\hline Accreditation process of oncology centres & -6.079 & -15.187 to 3.03 & 0.181 \\
\hline Access to multidisciplinary tumour boards & -14.464 & -28.038 to -0.889 & 0.038 \\
\hline Dermatologists responsible for organization of multidisciplinary tumour boards & -11.214 & -19.314 to -3.115 & 0.009 \\
\hline Medical oncologists' responsible for organization of multidisciplinary tumour boards & 2.526 & -7.24 to 12.292 & 0.598 \\
\hline \multicolumn{4}{|l|}{ Education of DER } \\
\hline Availability of subspecialization in oncology & -4.549 & -14.912 to 5.814 & 0.374 \\
\hline Availability of subspecialization in dermatologic surgery & -9.868 & -19.003 to -0.734 & 0.035 \\
\hline Availability of subspecialization in dermatopathology & -10.261 & -18.536 to -1.987 & 0.017 \\
\hline
\end{tabular}

*Estimated percentage of patients treated by different medical disciplines. 
Which medical specialties are performing skin cancer detection in your country and in what percentage?
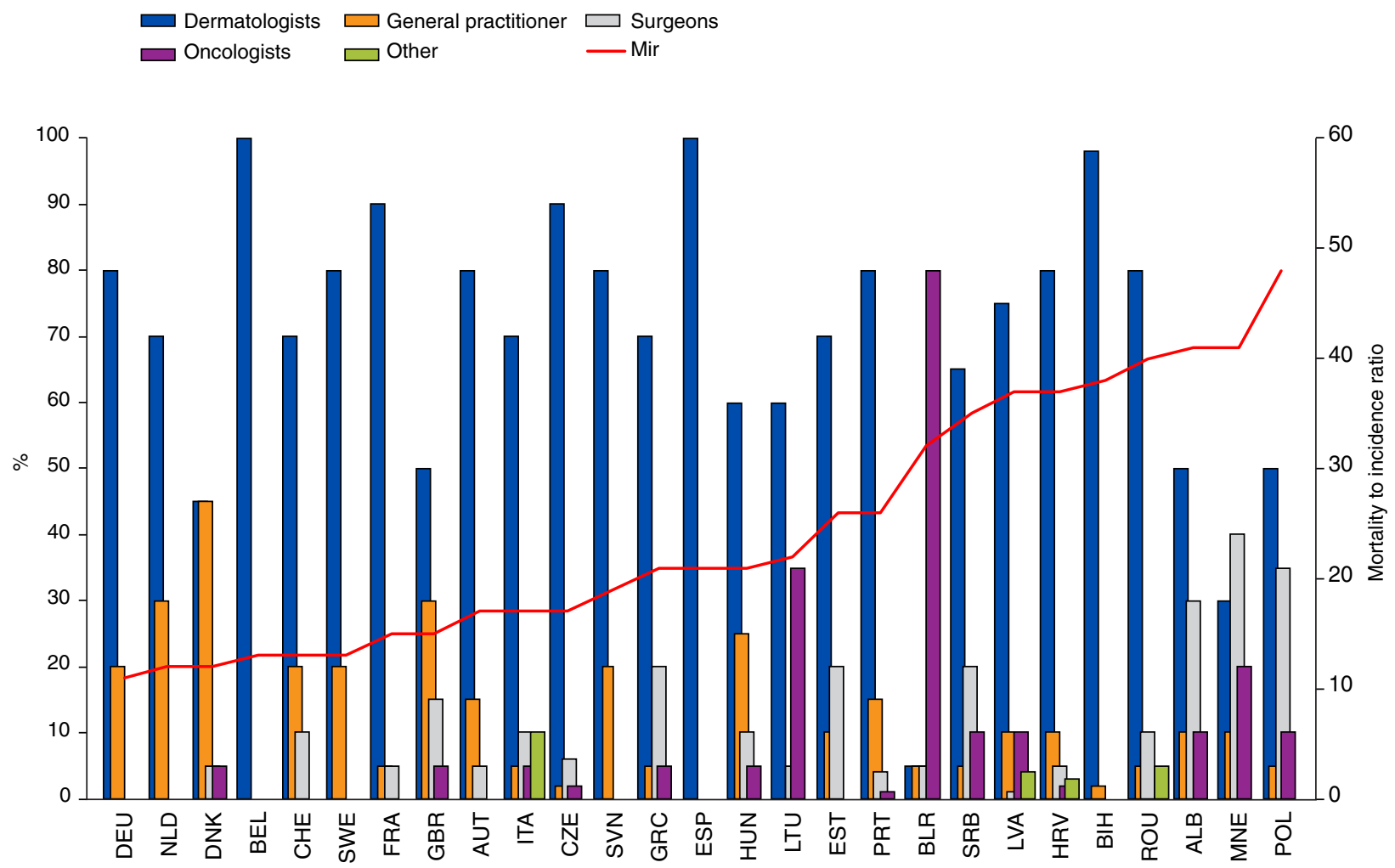

Figure 1 Medical disciplines involved in skin cancer detection and melanoma mortality-to-incidence ratio. ALB, Albania; AUT, Austria; BLR, Belarus; BEL, Belgium; BIH, Bosnia and Herzegovina; HRV, Croatia; CZE, Czech Republic; DNK, Denmark; EST, Estonia; FRA, France; DEU, Germany; GRC, Greece; HUN, Hungary; ITA, Italy; LVA, Latvia; LTU, Lithuania; MNE, Montenegro; NLD, the Netherlands; POL, Poland; PRT, Portugal; ROU, Romania; SRB, Serbia; SVN, Slovenia; ESP, Spain; SWE, Sweden; CHE, Switzerland; GBR, United Kingdom.

dermatopathology was found to be available in countries with a lower MIR. Also, skin cancer educational programmes for GPs were estimated to be available in $73 \%$ of the countries in NWE and $60 \%$ of those in SEE. Detailed analysis of dermato-oncology education in Europe was outside of the scope of this article and is planned for future studies.

A recent study found that not only late diagnosis but also less effective melanoma care can explain the persistent mortality disparities in Europe. ${ }^{31}$ In previous studies, quick access to pigmented lesion clinics was associated with higher rates and the earlier detection of melanoma. ${ }^{32-36}$ The current study found that melanoma care units with fast access upon primary care referrals (i.e. pigment lesion clinics) were established in approximately $92 \%$ of the countries in NWE and $53 \%$ of those in SEE. In previous studies, the presence of active MTBs led to better outcomes for cancer patients. ${ }^{37}$ The current study found that the existence of MTBs was also significantly correlated with a lower MIR, particularly in countries where DER were responsible for their organization. In addition, the MIR was lower in countries in which there was better access to diagnostic SLND, DER were involved in the follow-up of stage IB-IIC patients and DER and oncologists were responsible for the follow-up and systemic treatment of stage III and IV melanoma patients. Limitations in the prescription of systemic melanoma therapy or medical specialty-related restrictions in reimbursement seemed to be correlated with a higher MIR (Table 7). This highlights the need for integrated melanoma patient care from diagnosis through follow-up and treatment in centres of excellence where dermatology services could play the primary role, as indicated in the ECCO's essential requirements. ${ }^{7}$

Melanoma clinical registries, which are essential for the monitoring and quality control of diagnostic and treatment processes, were available in $54 \%$ of the countries (50\% in NWE and $57 \%$ in SEE). This was consistent with the population-based registry data generated from more comprehensive analyses. ${ }^{18,38,39}$ In recent decades, significant improvements have been made in 
registration, particularly with the establishment of the European Cancer Information System. However, there is still a need for the further development of population and melanoma clinical registries to improve quality control in melanoma care. ${ }^{18,38,39}$

A limitation of the current study is that it is an expert survey. A small proportion of the data were retrieved from the available national databases and national associations. The majority of the information was estimations from the current clinical practice of DER, ONC and SURG. However, these estimates correspond to the findings of previous studies. ${ }^{11,14}$ They can therefore be considered relevant, especially for the countries in which clinical data registries and healthcare audit data were not available.

The use of MIR as a proxy for fatality rates is not ideal. Indeed, the best-organized health systems tend to collect more accurate incidence data, while most of the countries do collect mortality data; thus, artificially higher ratios were found in the less organized health systems. Nevertheless, the MIR was found to be well correlated with the survival rates documented in the EUROCARE-5 study (Table S2). In the present study, many interacting variables were examined; thus, statistical reliability could not be achieved. However, some interesting trends were identified, and care was taken to avoid interpreting systematic associations and correlations as causal relationships.

The intention of the study was to provide an overview of the diagnosis and treatment of melanoma and skin cancer by the various medical specialties throughout Europe. In addition, the study aimed to estimate the extent to which the ECCO-recommended ideal melanoma care pathway had been implemented and to find exemplars that could guide improvements in the pathways in the various countries. In future studies, melanoma care pathways can be analysed on the basis of individual cases; however, national registries and/or European melanoma registry would need to be fully established in a majority of the countries to provide reliable data.

\section{Conclusions}

The incidence of skin cancer has been increasing throughout Europe; thus, healthcare systems should strive to fulfil the essential requirements for optimal care. The first estimates from this study suggest that skin cancer patients need integrated care that involves DER and GPs skilled in skin cancer detection, as well as SURG (e.g. dermatosurgeons, PS, ENT/MFS and surgical oncologists) in the initial surgical treatment. For lower-risk patients, optimal care also includes referrals to dermatologists for follow-up, and for patients with metastatic disease, it includes referrals to DER and oncologists for follow-up and treatment. The involvement of a higher number of professionals in patient care could contribute to lower mortality rates. It seems, that the greater involvement of DER who are highly trained in dermatoscopy, dermatosurgery and follow-up and treatment of melanoma may secure an optimal melanoma care pathway for patients. To confirm these estimates, melanoma care pathways can be analysed on the basis of individual cases and this should be explored in future studies.

\section{List of abbreviations:}

ECCO, European Cancer Organisation; EADO, European Association of Dermato-Oncology; NICE, UK National Institute for health and care excellence; GP, general practitioner; GNI, gross national income; HEPC, health expenditure per capita; UHC, universal health coverage; NWE, Northern and Western Europe; CEE, Central and Eastern Europe; SEE, Southern and Eastern Europe; MIR, mortality to incidence ratio; SLND, sentinel lymph node biopsy; DER, dermatologists; SURG, surgeons; ONC, medical oncologists; CDD, computerised digital dermatoscopy; RCM, reflectance confocal microscopy; OCT, optical coherence tomography; D, dermatologists; PS, plastic surgeons; MFS, maxillofacial surgeons; OS, oncological surgeons; GS, general surgeons; $\mathrm{P}$, pathologist; R, radiologist; RT, radiotherapist; SLND, sentinel lymph node dissection; MTB, multidisciplinary tumor board; ALB, Albania; AUT, Austria; BLR, Belarus; BEL, Belgium; BIH, Bosnia and Herzegovina; HRV, Croatia; CZE, Czech Republic; DNK, Denmark; EST, Estonia; FRA, France; DEU, Germany; GRC, Greece; HUN, Hungary; ITA, Italy; LVA, Latvia; LTU, Lithuania; MNE, Montenegro; NLD, the Netherlands; POL, Poland; PRT, Portugal; ROU, Romania; SRB, Serbia; SVN, Slovenia; ESP, Spain; SWE, Sweden; CHE, Switzerland; GBR, United Kingdom.

\section{Acknowledgements}

Statistical analysis and interpretation were completed with the assistance of Zoran Bukumiric, Associate Professor of Statistics, Institute of Medical Statistics, Faculty of Medicine, University of Belgrade, Serbia.

\section{References}

1 Sacchetto L, Zanetti R, Comber $\mathrm{H}$ et al. Trends in incidence of thick, thin and in situ melanoma in Europe. Eur J Cancer 2018; 92: 108-118.

2 Ferlay J, Colombet M, Soerjomataram I et al. Cancer incidence and mortality patterns in Europe: Estimates for 40 countries and 25 major cancers in 2018. Eur J Cancer 2018; 103: 356-387.

3 Stang A, Khil L, Kajüter $\mathrm{H}$ et al. Incidence and mortality for cutaneous squamous cell carcinoma: comparison across three continents. J Eur Acad Dermatol Venereol 2019; 33: 6-10.

4 Leiter U, Keim U, Eigentler T et al. Incidence, mortality, and trends of nonmelanoma skin cancer in Germany. J Invest Dermatol 2017; 137: 1860-1867.

5 Crocetti E, Mallone S, Robsahm TE et al. EUROCARE-5 Working Group: Survival of patients with skin melanoma in Europe increases further: results of the EUROCARE-5 study. Eur J Cancer 2015; 51: 2179-2190.

6 Forsea AM, Del Marmol V, Stratigos A, Geller AC. Melanoma prognosis in Europe:far from equal. Br J Dermatol 2014; 171: 179-182.

7 Wouters MW, Michielin O, Bastiaannet E et al. ECCO essential requirements for quality cancer care: melanoma. Crit Rev Oncol Hematol 2018; 122: 164-178.

8 NICE Pathways: managing melanoma NICE, 2020. Available at: http://pa thways.nice.org.uk/pathways/melanoma

9 Optimal cancer care pathway for people with melanoma. Cancer Council Australia. Available at: www.cancer.org.au/ocp.

10 Trakatelli M, Siskou S, Proby C et al. EPIDERM. The patient journey: a report of skin cancer care across Europe. Br J Dermatol 2012; 167: 43-52. 
11 DERMASurvey, European Dermatology Health Care Survey 2013. Institute for Health Services Research in Dermatology and Nursing. Available at: https://www.dermasurvey.eu/derma-survey/

12 Forsea AM, Tschandl P, Zalaudek I et al. The impact of dermoscopy on melanoma detection in the practice of dermatologists in Europe: results of a panEuropean survey. J Eur Acad Dermatol Venereol 2017; 31: 1148-1156.

13 Forsea AM, Tschandl P, del Marmol V, Zalaudek I, Soyer HP, Geller AC et al. Factors driving the use of dermoscopy in Europe: a pan-European survey. Br J Dermatol 2016; 175: 1329-1337.

14 Mathew A. Global survey of clinical oncology workforce. J Glob Oncol 2018; 4: 1-12.

15 Kandolf Sekulovic L, Guo J, Agarwala S et al. Access to innovative medicines for metastatic melanoma worldwide: Melanoma World Society and European Association of Dermato-oncology survey in 34 countries. Eur J Cancer 2018; 104: 201-209.

16 World Bank Group. Available at: http://www.worldbank.org/.

17 Healthcare personnel statistics - physicians. European Commission report, 2017. Available at: https://ec.europa.eu/eurostat.

18 European Cancer Information system - incidence and mortality estimates. Available at: https://ecis.jrc.ec.europa.eu/.

19 Mortality to incidence ratio. Available at: https://ncci.canceraustralia. gov.au/outcomes/mortality-incidence-ratio/mortality-incidence-ratio.

20 Ferlay J, Steliarova-Foucher E, Lortet-Tieulent J et al. Cancer incidence and mortality patterns in Europe: estimates for 40 countries in 2012. Eur J Cancer 2013; 49: 1374-1403.

21 Nikolaou V, Stratigos AJ. Emerging trends in the epidemiology of melanoma. Br J Dermatol 2014; 170: 11-19.

22 Barbaric J, de Vries E, Bray FI et al. Changing epidemiology of malignant cutaneous melanoma in Europe 1953-1997: rising trends in incidence and mortality but recent stabilizations in Western Europe and decreases in Scandinavia. Int J Cancer 2003; 107: 119-126.

23 Znaor A, van den Hurk C, Primic-Zakelj M et al. Cancer incidence and mortality patterns in Southeastern Europe in the last decade: gaps persist compared with the rest of Europe. Eur J Cancer 2013; 49: 1683-1691.

24 Hübner J, Hübner F, Terheyden P, Katalinic A. Turning point in skin cancer mortality: an analysis of the trend in Germany from 1998 to 2017. Hautarzt 2019; 70: 989-992.

25 Stratigos AJ, Forsea AM, van der Leest RJ et al. Euromelanoma: a dermatology-led European campaign against nonmelanoma skin cancer and cutaneous melanoma. Past, present and future. Br J Dermatol 2012; 167: 99-104.

26 Fleming NH, Grade MM, Bendavid E. Impact of primary care provider density on detection and diagnosis of cutaneous melanoma. PLoS One 2018; 13: e0200097.

27 Hopkins ZH, Moreno C, Carlisle R, Secrest AM. Melanoma prognosis in the United States: identifying barriers for improved care. J Am Acad Dermatol 2019; 80: 1256-1262.

28 Duarte AF, da Costa-Pereira A, Del-Marmol V, Correia O. Are general physicians prepared for struggling skin cancer? Cross-sectional study. $J$ Cancer Educ 2018; 33: 321-324.
29 Argenziano G, Giacomel J, Abramavicus A et al. Improving triage and management of patients with skin cancer: challenges and considerations for the future. Expert Rev Anticancer Ther 2012; 12: 609621.

30 Moscarella E, Lallas A, Longo C et al. Five-point checklist for skin cancer detection in primary care. G Ital Dermatol Venereol 2019; 154: $523-528$

31 Minicozzi P, Walsh PM, Sánchez MJ et al. EUROCARE-5 Working Group. Is low survival for cancer in Eastern Europe due principally to late stage at diagnosis? Eur J Cancer 2018; 93: 127-137.

32 Lipworth AD, Park JM, Trefrey BL et al. Urgent access to a specialty care melanoma clinic is associated with a higher rate of melanoma detection. J Am Acad Dermatol 2011; 64: 1060-1067.

33 Pacifico MD, Pearl RA, Grover R. The UK government two-week rule and its impact on melanoma prognosis: an evidence-based study. Ann $R$ Coll Surg Engl 2007; 89: 609-615.

34 Pacifico MD, Grover R, Sanders R. Use of an early-detection strategy to improve disease control in melanoma patients. Br J Plast Surg 2004; 57: 105-111.

35 Lynch M, Tierney E, Roche L et al. Melanoma diagnosis and management after the introduction of a pigmented lesion clinic in the Mid-West of Ireland. Ir J Med Sci 2017; 186: 671-675.

36 Carli P, De Giorgi V, Nardini P et al. Melanoma detection rate and concordance between self-skin examination and clinical evaluation in patients attending a pigmented lesion clinic in Italy. Br J Dermatol 2002; 146: $261-266$

37 Prades J, Remue E, van Hoof E, Borras JM. Is it worth reorganising cancer services on the basis of multidisciplinary teams (MDTs)? A systematic review of the objectives and organisation of MDTs and their impact on patient outcomes. Health Policy 2015; 119: 464-474.

38 Forsea AM. Cancer registries in Europe - going forward is the only option. Ecancermedicalscience. 2016; 10: 641.

39 European Network of Cancer Registries. Available at: https://www.enc r.eu.

\section{Supporting information}

Additional Supporting Information may be found in the online version of this article:

Table S1 Questionnaire used in the study.

Table S2 Correlation of mortality-to-incidence ratio with 1-year and 5-year survival.

Table S3 Correlation of health expenditure per capita to melanoma care pathway components. 